\title{
THE QSO HE 0450-2958: SCANTILY DRESSED OR HEAVILY ROBED? A NORMAL QUASAR AS PART OF AN
} UNUSUAL ULIRG

\author{
Knud Jahnke ${ }^{1}$, David Elbaz ${ }^{2}$, Eric Pantin ${ }^{2}$, Asmus Böhm $^{3,4}$, Lutz Wisotzki $^{3}$, Geraldine Letawe $^{5}$, Virginie Chantry ${ }^{5}$, \\ AND PierRe-Olivier Lagage ${ }^{2}$ \\ ${ }^{1}$ Max-Planck-Institut für Astronomie, Königstuhl 17, D-69117 Heidelberg, Germany; jahnke@ mpia.de \\ ${ }^{2}$ CEA Saclay/Service d'Astrophysique, Laboratoire AIM, CEA/DSM/IRFU-CNRS-Université Paris Diderot, F-91191 Gif-sur-Yvette Cédex, France \\ 3 Astrophysikalisches Institut Potsdam, An der Sternwarte 16, D-14482 Potsdam, Germany \\ ${ }^{4}$ Institut für Astro- und Teilchenphysik, Universität Innsbruck, Technikerstraße 25/8, A-6020 Innsbruck, Austria \\ ${ }^{5}$ Institut d'Astrophysique et Géophysique, Université de Liège, Allée du 6 Août, 17 Sart Tilman (Bat. B5C), B-4000 Liège, Belgium \\ Received 2008 December 3; accepted 2009 June 9; published 2009 July 16
}

\begin{abstract}
The luminous $z=0.286$ quasar HE 0450-2958 is interacting with a companion galaxy at $6.5 \mathrm{kpc}$ distance and the whole system radiates in the infrared (IR) at the level of an ultraluminous infrared galaxy (ULIRG). A so far undetected host galaxy triggered the hypothesis of a mostly "naked" black hole (BH) ejected from the companion by three-body interaction. We present new Hubble Space Telescope (HST)/NICMOS $1.6 \mu$ m imaging data at 0'.1 resolution and VLT/VISIR $11.3 \mu \mathrm{m}$ images at $0^{\prime \prime} .35$ resolution that are for the first time resolving the system in the near- and mid-infrared. We combine these data with existing optical $H S T$ and CO maps. (1) At $1.6 \mu \mathrm{m}$ we find an extension NE of the quasar nucleus that is likely a part of the host galaxy, though not its main body. If true, a combination with upper limits on a main body co-centered with the quasar brackets the host-galaxy luminosity to within a factor of $\sim 4$ and places HE 0450-2958 directly onto the $M_{\mathrm{BH}}-M_{\text {bulge }}$ relation for nearby galaxies. (2) A dust-free line of sight to the quasar suggests a low dust obscuration of the host galaxy, but the formal upper limit for star formation (SF) lies at $60 M_{\odot} \mathrm{yr}^{-1}$. HE 0450-2958 is consistent with lying at the high-luminosity end of Narrow-Line Seyfert 1 Galaxies, and more exotic explanations like a "naked quasar" are unlikely. (3) All $11.3 \mu \mathrm{m}$ radiation in the system is emitted by the quasar nucleus. It has warm ULIRG-strength IR emission powered by $\mathrm{BH}$ accretion and is radiating at super-Eddington rate, $L / L_{\mathrm{Edd}}=6.2_{-1.8}^{+3.8}$, or $12 M_{\odot} \mathrm{yr}^{-1}$. (4) The companion galaxy is covered in optically thick dust and is not a collisional ring galaxy. It emits in the far-infrared at ULIRG strength, powered by Arp220-like SF (strong starburst-like). An M82-like SED is ruled out. (5) With its BH accretion rate, HE 0450-2958 produces not enough new stars to maintain its position on the $M_{\mathrm{BH}}-M_{\text {bulge }}$ relation, and SF and BH accretion are spatially disjoint. This relation can either only be maintained averaging over a longer timescale $(\lesssim 500 \mathrm{Myr})$ and/or the bulge has to grow by redistribution of pre-existing stars. (6) Systems similar to HE 0450-2958 with spatially disjoint ULIRG-strength star formation and quasar activity might be common at high redshifts but at $z<0.43$ we only find $<4 \%(3 / 77)$ candidates for a similar configuration.
\end{abstract}

Key words: galaxies: active - galaxies: evolution - galaxies: interactions - galaxies: starburst - infrared: galaxies - quasars: individual (HE0450-2958)

\section{INTRODUCTION}

In the current framework of galaxy evolution, galaxies, and black holes (BHs) are intimately coupled in their formation and evolution. The masses of galactic bulges and their central BHs in the local universe follow a tight relation (e.g., Häring \& Rix 2004) with only 0.3 dex scatter. Currently it is not clear how this relation comes about and if and how it evolved over the last $13 \mathrm{Gyr}$, but basically all semi-analytic models now include feedback from active galactic nuclei (AGNs) as a key ingredient to acquire consensus with observations (e.g., Hopkins et al. 2006; Somerville et al. 2008). In these models, it is assumed that $\mathrm{BH}$ growth by accretion and energetic re-emission from the ignited AGNs back into the galaxy can form a self regulating feedback chain. This feedback loop can potentially regulate or possibly also truncate star formation (SF) and in this process create and maintain the red/blue color-magnitude bimodality of galaxies. In this light, any galaxy with an abnormal deviation from the $M_{\mathrm{BH}}-M_{\text {bulge }}$ relation will be an important laboratory for understanding the coupling mechanisms of $\mathrm{BH}$ and bulge growth. It will set observational limits for these models, and constrain the timelines and required physics involved.
Since the early work by Bahcall et al. (1994, 1995) on QSO host galaxies with the Hubble Space Telescope (HST) and the subsequently resolved dispute about putatively "naked" QSOs (McLeod \& Rieke 1995), no cases for QSOs without surrounding host galaxies were found-when detection limits were correctly interpreted. Only recently the QSO HE 0450 2958 renewed the discussion, when Magain et al. (2005) made a case for a six times too faint upper limit of the host galaxy of HE 0450-2958 with respect to the $M_{\mathrm{BH}}-M_{\text {bulge }}$ relation. In light of a number of competing explanations for this, the nature of the HE 0450-2958 system needs to be settled.

The QSO HE 0450-2958 (a.k.a. IRAS 04505-2958) at a redshift of $z=0.286$ was discovered by Low et al. (1988) as a warm IRAS source. HE 0450-2958 is a radio-quiet quasar, with a distorted companion galaxy at 1".5 (=6.5 kpc) distance at the same redshift, likely in direct interaction with the QSO (Canalizo \& Stockton 2001). The combined system shows an infrared (IR) luminosity of an ultraluminous infrared galaxy (ULIRG, $L_{\mathrm{IR}}>10^{12} L_{\odot}$ ).

HE 0450-2958 was observed with the HST and its WFPC2 camera (Boyce et al. 1996) in F702W ( $=R$ band) and ACS camera (Magain et al. 2005) in F606W ( $=V$ band), both 
observations did not allow to detect a host galaxy centered on the quasar position within their limits (Figure 1, left column). Magain et al. (2005) estimated an expected host-galaxy brightness if HE 0450-2958 was a normal QSO system that obeyed the $M_{\mathrm{BH}}-M_{\text {bulge }}$ relation in the local universe and given a $\mathrm{BH}$ mass estimate or luminosity of the QSO. They concluded that the ACS F606W detection limits were six times fainter than the expected value for the host galaxy, which qualified HE 04502958 to be very unusual.

Magain et al. (2005) sparked a flurry of subsequent papers to explain the undetected host galaxy to $\mathrm{BH}$ relation. Over time, three different alternative explanations have been put forward and were substantiated:

1. HE 0450-2958 is a normal QSO nucleus, but with a massive $\mathrm{BH}$ residing in an under-massive host galaxy. The system is lying substantially off the local $M_{\mathrm{BH}}-M_{\text {bulge }}$ relation; the host galaxy possibly hides just below the F606W detection limit (Magain et al. 2005).

2. The host galaxy is actually absent, HE 0450-2958 is a truly "naked" QSO, by means of a $\mathrm{BH}$ ejection event in a gravitational three-body interaction or gravitational recoil following the merger of HE 0450-2958 with the companion galaxy (Hoffman \& Loeb 2006; Haehnelt et al. 2006; Bonning et al. 2007).

3. The original $\mathrm{BH}$ mass estimate was too high (Merritt et al. 2006; Kim et al. 2007; Letawe et al. 2007) and is in fact $\sim 10$ times lower. With comparably narrow $\left(\sim 1500 \mathrm{~km} \mathrm{~s}^{-1}\right.$ FWHM) broad QSO emission lines the QSO could be the high-luminosity analog of the class of narrow-line Seyfert 1 galaxies (NLSy1). The host galaxy could be normal for the BH mass and be absolutely consistent with the ACS upper limits.

In this article, we present new data initially motivated by the still undetected host galaxy and by the possibility that the host galaxy might be obscured by substantial amounts of dust. We want to investigate the overall cool and warm dust properties of the system, using new near-infrared (NIR) and mid-infrared (MIR) images. The F606W ACS band is strongly susceptible to dust attenuation, and dust could have prevented the detection of the host galaxy in the optical. With new NIR data, we look at a substantially more transparent wavelength.

At the same time the new IR data is meant to localize the source(s) of the ULIRG emission. Three components are candidates for this: the active galactic nucleus, the host galaxy, and the companion galaxy. Our NIR data allow to trace SF and the MIR image traces the hot dust in the system. We present the new data and interpret them in the view of the so far collected knowledge from X-ray to radio wavelengths that was built up since the article of Magain et al. (2005).

Throughout we will use Vega zero points and a cosmology of $h=H_{0} /\left(100 \mathrm{~km} \mathrm{~s}^{-1} \mathrm{Mpc}^{-1}\right)=0.7, \Omega_{M}=0.3$, and $\Omega_{\Lambda}=0.7$, corresponding to a distance modulus of 40.84 for $z=0.286$ and linear scales of $4.312 \mathrm{kpc} \operatorname{arcsec}^{-1}$.

\section{THE IR ANGLE}

Up to now the only existing IR observations on HE 04502958 were from the 2MASS survey in the NIR $J, H$, and $K$ bands at $\sim 4^{\prime \prime}$ resolution, and in the MIR from the IRAS mission (de Grijp et al. 1987; Low et al. 1988) at 12, 25, 60, and $100 \mu \mathrm{m}$ with about $4^{\prime}$ resolution. Both surveys do not resolve the different individual components of the system (QSO, companion galaxy, foreground star). De Grijp et al. (1987) noted that the
HE 0450-2958 system is showing the MIR/FIR luminosities of a ULIRG system, but it was not clear which components of the system are responsible for this emission due to the coarse IRAS resolution. We want to localize the dust emission in two ways: (a) a direct observation of the hot dust component at $8.9 \mu \mathrm{m}$ (rest frame) with the VISIR imager at the ESO VLT. (b) A localization of dust in general by combining new HST NIR and the existing ACS optical data. For this purpose we obtained $H S T$ NIC2 imaging in the rest-frame $J$ band at $\sim 1.3 \mu \mathrm{m}$.

\subsection{VISIR 11.3 $\mu \mathrm{m}$ Imaging Data}

In the NIR and MIR, the HE 0450-2958 system clearly has a spectral energy distribution (SED) that is composed of more than a single component: in Figure 2 we model the IRAS and VISIR flux densities with a composite SED of a quasar plus a starforming component. For the quasar we test the median and 68 percentile reddest quasar SED from Elvis et al. (1994), and for the star-forming component an Arp220-like starbursting SED, but we also tried a medium SF M82 SED, both from Elbaz et al. (2002). The median quasar SED plus Arp220 can reproduce the data at all wavelengths, except at observed $25 \mu \mathrm{m}$, where it leaves a small mismatch. The 68 percentile reddest SED on the other hand creates a perfect match also there. For both cases, the flux predicted for the companion galaxy at $11.3 \mu \mathrm{m}$ lies below the detection limit as observed. Milder, M82-like SF can be ruled out on the same basis, as it predicts a detection of the companion also at $11.3 \mu \mathrm{m}$-both with the information from the MIR, as well as when extrapolating the observed $H$-band flux.

Papadopoulos et al. (2008) matched a simple model of two blackbody emission curves to the four IRAS points, yielding a cool dust component heated by SF and a warm dust component which can be attributed to intense AGN emission (see Section 4.1). While it is not possible to spatially resolve the system at FIR wavelengths with current telescopes, we aim for the highest wavelength where this is currently possible, in order to localize the warm emission component and test whether this comes solely from the (optically visible) QSO or from extra sources.

The observations were performed using VISIR, the ESO/ VLT MIR imager and spectrograph mounted on unit 3 of the VLT (Melipal). VISIR gives a pixel size of 0.'075 and a total field-of-view of $19^{\prime \prime} .2$. The diffraction limited resolution is 0.35 FWHM. Standard "chopping and nodding" MIR observational technique was used to suppress the background dominating at these wavelength. All the observations were interlaced with standard star observations of HD 29085 (4.45 Jy) and HD 41047 (7.21 Jy). The estimated sensitivity was $4 \mathrm{mJy} / 10 \sigma / 1 \mathrm{~h}$.

Imaging data were obtained on 2005 December 12 in service observing mode, through the PAH2 filter centered on $11.3 \mu \mathrm{m}$ having a half-band width of $0.6 \mu \mathrm{m}$. Weather conditions were very good, optical seeing was below $1^{\prime \prime}$, and the object was observed always at an airmass of 1.15 , which resulted in a diffraction limited image of 0 '.35 resolution. Chopping/nodding parameters were $8^{\prime \prime} / 8^{\prime \prime}$ and $0.25 \mathrm{~Hz} / 0.033 \mathrm{~Hz}$. The total time spent on-source was 1623 s. The data were reduced using a dedicated pipeline written in IDL, which does the chopping/ nodding correction and removes the spurious stripes due to detector instabilities (Pantin et al. 2008). The reduced data were finally flux-calibrated using the two reference stars as photometric calibrators. The error on the photometry due to variations of the atmospheric transmission are estimated to be less than $2 \%(3 \sigma)$. 

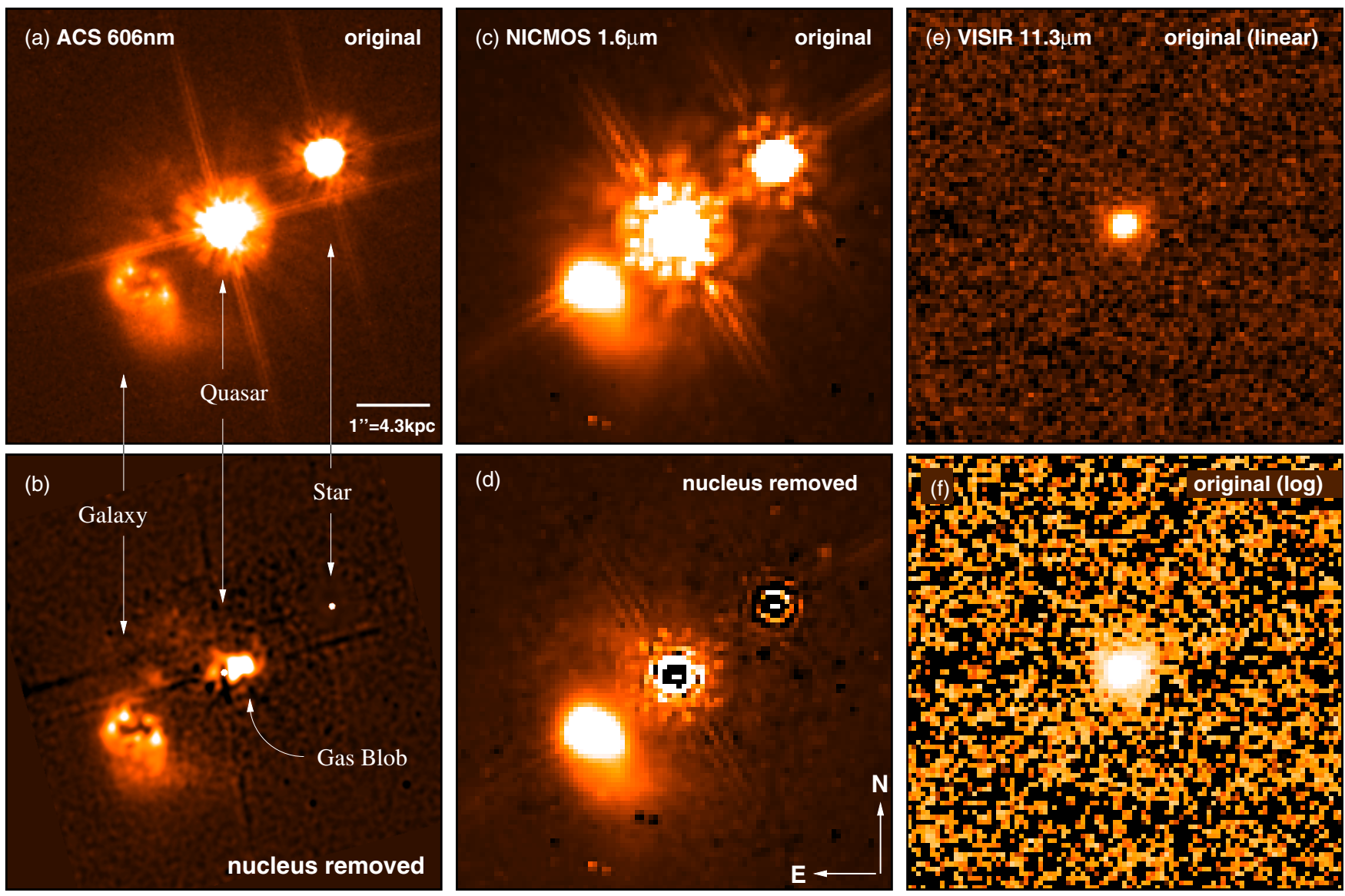

Figure 1. HE 0450-2958 system as seen in the existing optical images, and our new NIR and MIR data with the same scale and orientation (field size is $7^{\prime \prime}$, N is up and $\mathrm{E}$ to the left). Shown are (a) the original ACS HRC image in the $V$ band (F606W) by Magain et al. (2005), (b) the deconvolved ACS HRC image, (c) the original NICMOS $H$ band (F160W), (d) the NICMOS image after image decomposition and subtraction of the nuclear component and foreground star, and the VISIR PAH2 filter image at $11.3 \mu \mathrm{m}$ (e) in linear stretch and (f) in logarithmic stretch. See Figure 3 for more NICMOS images. The VISIR image (e+f) is diffraction limited but shows a single unresolved $62.5 \mathrm{mJy}$ point source, the AGN. No other source in the field is detectable above a point-source flux density of $3 \mathrm{mJy}(5 \sigma)$, the companion galaxy is also undetected. In the ACS image (b) the PSF-distributed flux of the point like QSO nucleus and foreground star have been condensed into the two white points. The "Blob" 0".5 from the QSO is made only of emission line light (Letawe et al. 2008) - and thus not visible in the NIR since we have no similar line in the F160W bandpass. The companion galaxy $1 . .5(=6.5 \mathrm{kpc})$ to the $\mathrm{SW}$ has $M_{V}=-23$ (Magain et al. 2005). It has a complex structure, but as a difference to the optical it is clearly peaked toward the center in the NIR. Toward the SW of the companion the tidal arm described by Canalizo \& Stockton (2001) is visible.

\subsection{NICMOS H-band Imaging Data}

The ACS $V$-band is too blue to penetrate any substantial amount of dust. With the scenario of a dust enshrouded host galaxy in mind, we acquired new HST NICMOS data (NIC2 with $0^{\prime \prime} .075$ plate scale) in the F160W H-band (program \#10797, cycle 15) to reduce the dust attenuation by a factor of 3.5 in magnitude space.

A total of $5204 \mathrm{~s}$ integration on target was forcedly split into two observation attempts due to telescope problems, and carried out in 2006 and 2007 July. These yielded two sets of data with $2602 \mathrm{~s}$ integration each, but slightly different orientations. In order to minimize chromatic effects, we also observed a point-spread function (PSF) calibrator star (EIS J033259.33-274638.5) with the SED-characteristics over the F160W filter bandpass similar to a mean QSO template. We do not know the actual SED of HE 0450-2958 itself, as no NIR imaging or spectroscopic data of the system with high enough spatial resolution exist to date. As the stellar type yielding the likely most similar PSF we found K4III, by comparing the PSFs predicted by the TinyTim package (J. Krist, http://www.stsci.edu/software/tinytim). The only cataloged stars faint enough to not immediately saturate were observed by the ESO Imaging Survey (EIS, Groenewegen et al. 2002) located in the E-CDFS, and had to be observed at six months distance in time to HE 0450-2958. Since we also want to minimize the PSF variation due to differences in observing strategy, we applied the same dither patterns for both HE 04502958 and the PSF star. Due to the absolute pointing accuracy of HST the centroid location of the star relative to the chip is shifted about 15 pixels (1"1) from the QSO centroid toward the companion galaxy.

Data reduction and combination of the individual frames were carried out using a mix of STScI pipeline data products, pyraf, and our own procedures in MIDAS and Fortran. The resulting image is shown together with the analysis in Figure 3(a). Two parts of the team analyzed the combined images in complementary ways, by decomposition of the components using two-dimensional modeling and by image deconvolution.

\subsubsection{Uncertainty in the PSF}

In order to detect a putative faint host galaxy underneath the bright QSO nucleus, we require a precise knowledge of the PSF. The PSF will vary spatially, with the energy distribution in the filter as well as temporally, with a changing effective focus of the telescope due to changing thermal history. 


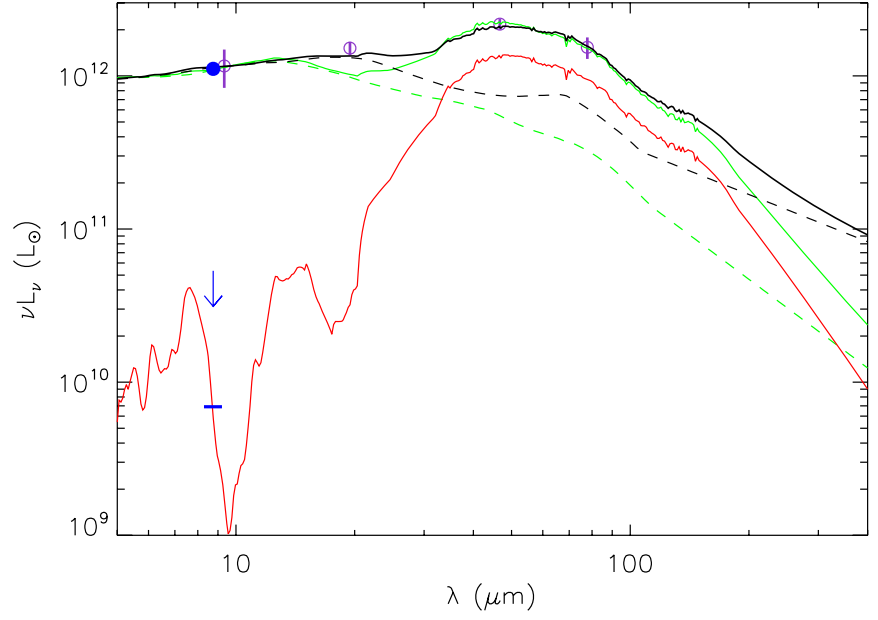

Figure 2. SED of HE 0450-2958 in the MIR: shown are the IRAS flux density measurements from de Grijp et al. (1987; open circles), our VISIR data point (filled circle) and upper limit on the companion galaxy (arrow) and overlaid composite AGN plus starburst SEDs (lines). For the quasar nucleus we use the median (green dashed line) and 68 percentile reddest SEDs (black dashed line) from Elvis et al. (1994), the starburst (red solid line) is a model for Arp220 by Elbaz et al. (2002). The median quasar plus Arp220 SED (green solid line) can explain the data except for a slightly too low value at the observed $25 \mu \mathrm{m}$ point, but with the 68 percentile SED (black solid line) the match is perfect. The predicted flux of the companion galaxy where the SF of the system is located (bar) lies below our detection limit, consistent with the data. Milder SF templates as, e.g., M82 can be ruled out, since they predict too high fluxes for the companion-also from the observed $H$-band data-which should be visible in the VISIR image.

We opt for a double approach: first, we observe the separate PSF star with the properties described in the last section (and see Figure 3(b)). Second, we also have the foreground star available that is located at $1 . .8$ distance from the QSO to the NW. It is classified as a $\mathrm{G}$ star (Low et al. 1989). Its on-chip distance to the QSO will leave only room for small spatial variations, but its SED in the $H$ band likely will not perfectly match the SED of the QSO.

It is difficult to assess the PSF uncertainty at the position of the QSO. In principle we have a combined effect of color, spatial, and temporal variation, but only one bit of information: the difference between the foreground star and the PSF star. We, thus, model the expected difference in the shape of these two stars with TinyTim and then compare their actual observed shapes. This shows that the foreground star should be slightly narrower than the observed PSF star, which is consistent with PSF star's later, redder spectral type and an increase of PSF width with wavelength. We observe this effect also in the data, however somewhat stronger. A temporal variation can thus not be separated and ruled out.

In any case, we conclude that the PSF star is wider and thus will yield more conservative (=fainter) estimates for a QSO host galaxy, while in case of a non-detection the foreground star will yield brighter upper limits.

For two-dimensional modeling of the system we use GALFIT (Peng et al. 2002). In order to quantify the PSF uncertainty for this process, we first let GALFIT fit a single point source, represented by the PSF star, to the foreground star. In this process, we use an error map created from the data itself and we add the sky as a free parameter. We minimize the influence of the nearby QSO on the foreground star by first fitting the former with a single point source as well, removing its modeled contribution, and mask out the remaining residuals starting at
0 .'9 from the star. The PSF created in this way is shown in Figure 3(c). This image is fed into the modeling process of the PSF star, or later the QSO/host/companion system.

The residual flux in this process is of the order of $3 \%$ of the total, inside the 0.5 radius aperture where most apparent residuals are located, the absolute value of the residuals in the same region is $14 \%$. This means that it will be generally impossible to detect any host galaxy of less than $3 \%$ of the total flux of the QSO, and it will even be difficult to isolate a somewhat brighter smooth galaxy in the non-smooth residuals. This level of residuals is consistent with experience from the HST ACS camera, where we find that due to PSF uncertainties $5 \%$ of the total flux is the approximate detection limit for faint host galaxies (Jahnke et al. 2004b, K. Jahnke et al. 2009, in preparation). Including the structured PSF residuals we will only consider a host-galaxy component as significant if it has clearly more than $3 \%-5 \%$ of residual flux inside an 0.5 radius of the QSO, or that shows up as a non-co-centric structure above the noise outside this region.

In absolute magnitudes and related to the QSO these limits correspond to the following: inside an 0.5 radius of the QSO we can hide a galaxy co-centric with the QSO of at least $M_{H} \sim-24.7$ (for the $3 \%$ case) or $M_{H} \sim-25.2$ (for 5\%).

\section{RESULTS}

\subsection{VISIR}

We detect a single unresolved point source in the VISIR field-of-view with a flux density $62.5 \mathrm{mJy}$ at observed 11.3 $\mu \mathrm{m}$ (Figure 1, right column). This compares to $69.3 \mathrm{mJy}$ in the IRAS $12 \mu \mathrm{m}$ channel. There is no second source detected in the field down to a point-source sensitivity of at least $3 \mathrm{mJy}$ at the $5 \sigma$ level. Extended sources of the visual size of the companion galaxy have a $5 \sigma$ detection limit of $5.5 \mathrm{mJy}$.

With only one source in the total 19.2 VISIR field three optical sources have in principle to be considered as potential counterparts: The QSO nucleus, the companion galaxy, and the foreground star. However, the star is a G spectral type and can thus be safely ruled out.

We find that the initial position of the MIR point source as recorded in the VISIR image header comes to lie between the QSO and the companion, somewhat closer to the QSO. To clarify this we conducted an analysis of the pointing accuracy of VISIR testing the astrometry of a number of reference stars observed with VISIR at different epochs. The two results are: (1) in all cases the offset between targeted and effective R.A., decl. is less than $1^{\prime \prime}$ rms, but (2) there is a systematic offset of $0.15 \mathrm{~s}$ in R.A. recorded in the fits header, so the true positions need to be corrected by $-0.15 \mathrm{~s}$ in R.A. This correction places the MIR point source exactly onto the locus of the QSO in the HST ACS images. It is, thus, clearly the QSO nucleus that is responsible for all of the $11.3 \mu \mathrm{m}$ emission.

\subsection{NICMOS}

\subsubsection{Host Galaxy}

To extract information on the host galaxy, we use three different methods to remove the flux contribution from the QSO nucleus. First, we make a model-independent test for obvious extended emission: in a simple peak subtraction we remove a PSF from the QSO, scaled to the total flux inside two pixel radius around the QSO center. This is a robust approach that is independent of specific model assumptions and quite insensitive 

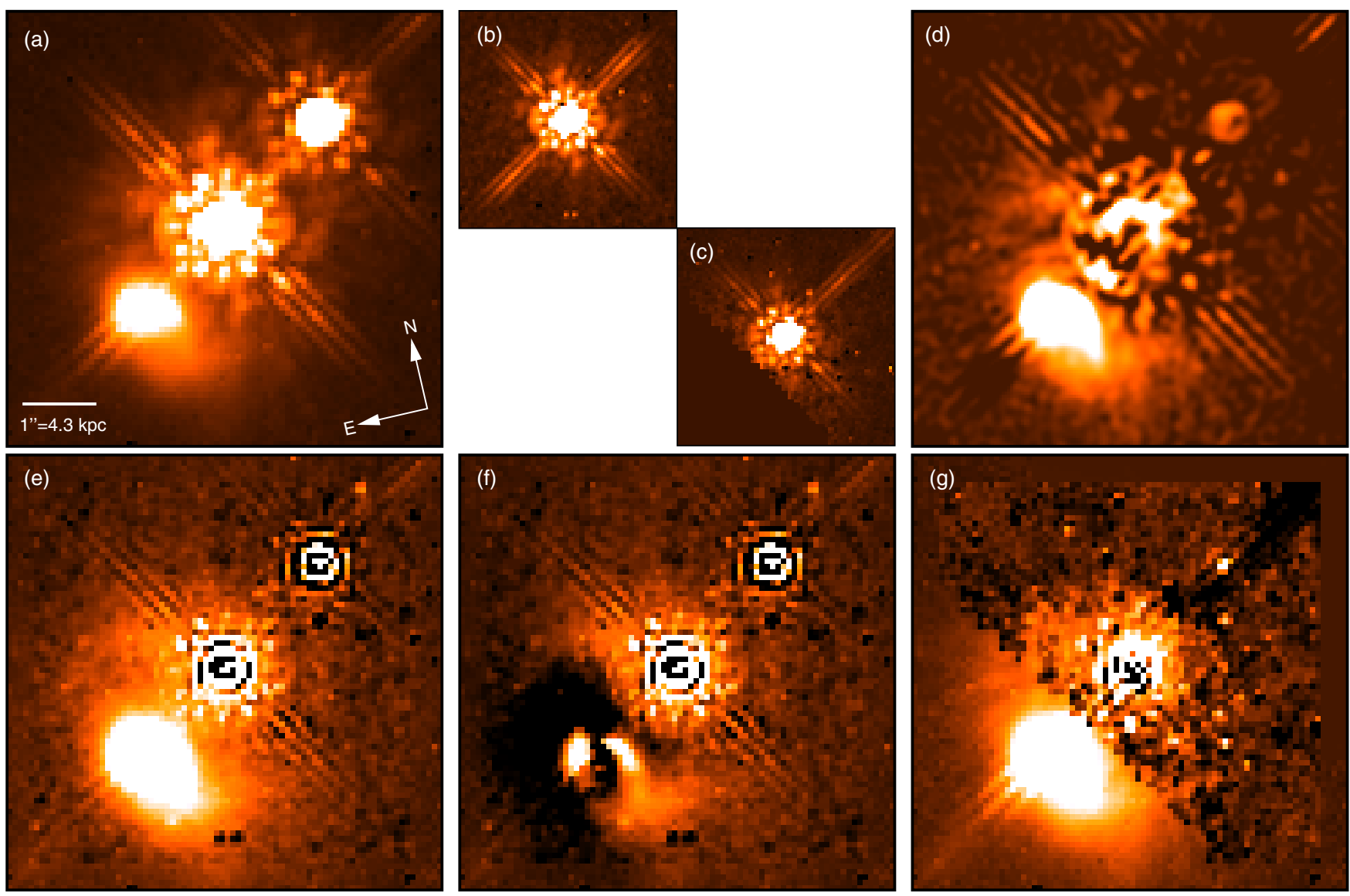

Figure 3. NICMOS $H$-band data. Shown are (a) original observed image, (b) the separately observed PSF star EIS J033259.33-274638.5 and (c) cleaned and masked foreground star used as alternative PSF. The system is further shown after nucleus removal: (d) System after MCS-deconvolution and (e-g) after 2dim-modeling with GALFIT. Deconvolution uses as a PSF an envelope from the PSF star (b) with a core from the companion star (c). For GALFIT modeling, QSO and foreground star are represented with one point source each, using either $(\mathrm{e}+\mathrm{f})$ the PSF star as a PSF or the $(\mathrm{g})$ foreground star. The companion is neither represented well with one nor two Sérsic components as seen in (f) for the otherwise same setup as (e), where the companion was not modeled. No significant host galaxy is seen cocentric with the QSO center. However a faint emission is visible at $\sim 0^{\prime \prime} 6-1^{\prime \prime} .5$ to the NE (see Figure 4 for more details). The displayed image size is 80 NIC 2 pixels of $0{ }^{\prime \prime} 075$ size, i.e., $6^{\prime \prime}$.

to the noise distribution in the image (Jahnke et al. 2004b). As a result, the peak subtracted image shows no obvious extended residual, i.e., host galaxy, centered on the QSO, when using the PSF star as PSF.

As a second step, on the one hand we use GALFIT to model the two-dimensional light distribution of the HE 0450-2958 system and decompose it into different morphological components. On the other hand we use the MCS deconvolution method (Magain et al. 1998) to mathematically deconvolve the system to a well defined and narrower PSF. The procedure we follow is based on the one described in Chantry \& Magain (2007). For GALFIT we use the two empirical PSFs, for MCS deconvolution we construct a number of combinations of empirical PSF and TinyTim models including very red dust-like SED components.

While these two approaches are complementary in method, their results agree as can be seen in Figure 3: the inner part of the QSO inside of 0'.5 radius is consistent with a point source within the PSF uncertainties, but there is extra flux present outside of this radius. The structure of the PSF removal or deconvolution residuals points to a substantial mismatch between shape of the QSO nucleus and the separately observed PSF star, but also to too simple models of TinyTim. In order to remove obvious residual PSF structure a very red SED needs to be assumed, which at this point cannot be discriminated from a marginally resolved red component on top of the AGN point source. However, in light of the non-average properties of this QSO, a mean QSO SED is also not expected.

In the following, we present our results in more detail and focus on the GALFIT results, since it allows a more direct estimate of the significance of detected structures. A comparison of the original and point-source-removed images in the optical and NIR, and the MIR image is shown in Figure 1.

We use GALFIT to perform a number of different model fits. In all of them the companion star and QSO nucleus are described by a pure point source, while the companion galaxy is fit with one or two Sérsic ${ }^{6}$ components with free axis ratio, or left unmodeled. We also attempt to add another Sérsic component for the putative host galaxy. We always leave the Sérsic parameter $n$ free, although the companion galaxy is too complex and the putative host galaxy too faint for $n$ to be interpreted physically.

With the PSF star used as PSF GALFIT finds a result consistent with the peak subtraction. A positive residual of $H \sim 17.7$ inside a $\sim 1^{\prime \prime}$ radius aperture has a flux below $2 \%$ of the 13.05 mag of the QSO itself (see Figure 3(e+f)). Even though we choose an aperture larger than in our calculation in Section 2.2.1, we receive a value far below our significance limit, so no significant co-centered host galaxy is seen in this way.

\footnotetext{
6 The Sérsic profile (Sérsic 1968) is a generalized galaxy profile with variable wing strength, set by the "Sérsic-parameter" $n$. It reverts to an exponential disk profile typical for spiral galaxies for $n=1$ and for $n=4$ it becomes a de Vaucouleurs profile found for many elliptical galaxies.
} 


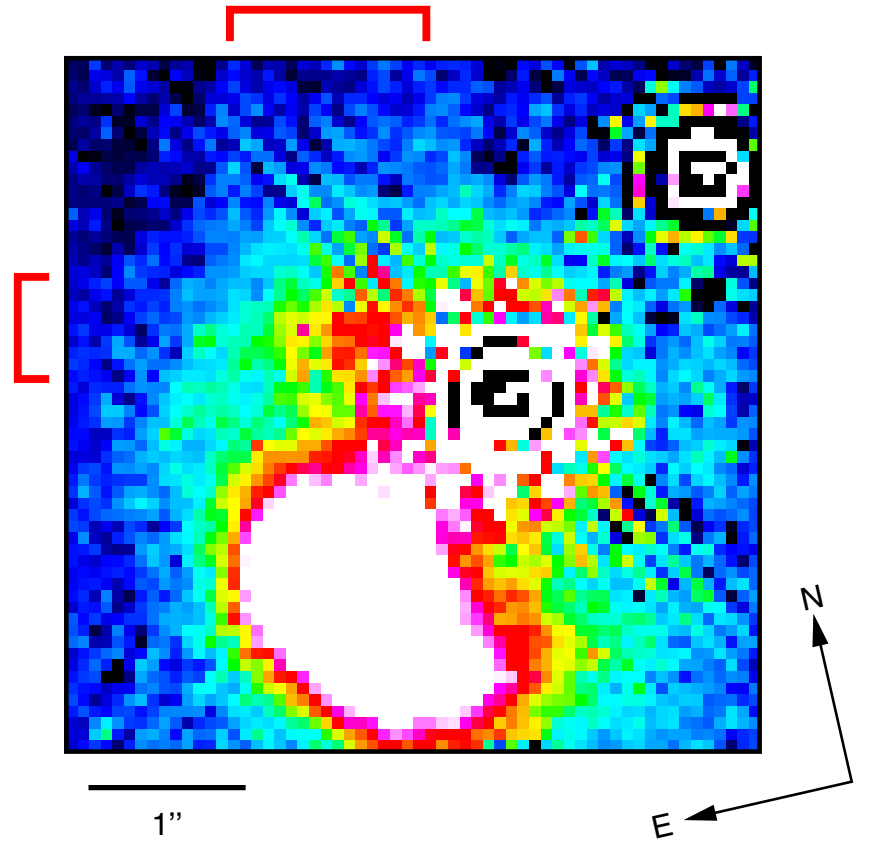

Figure 4. Slight zoom into the inner region of HE 0450-2958 to show the newly found "NE-extension" of the QSO. We removed the star and the QSO using the PSF star as PSF. An extension to the NE is visible (marked with red brackets) at a distance of $0 .{ }^{\prime} 6-1{ }^{\prime \prime} .5$ that is clearly not due to PSF residuals-a very similar result is seen when using the narrower foreground star as PSF (Figure 3(e)), or MCS deconvolution (Figure 3(d)). This structure is disjoint from the companion galaxy so very likely belongs to the QSO host galaxy itself. The estimated brightness is $H=18.8$. The image size is $4^{\prime \prime} .5$ on the side.

If we use the foreground star as PSF (Figure 3(g)) we find-as expected - a residual flux that is slightly higher than before, and consistent values for two different approaches: first, for a pure PSF fit to the QSO location, integrating the flux of the residual within a $1^{\prime \prime}$ radius aperture around the QSO, except along the SE-NW axis where we expect residual flux from foreground star and companion galaxy. Second, we get a similar flux for a fitted additional host-galaxy Sérsic component.

These two approaches yield a magnitude of $H \sim 15.8$ and 16.2 , respectively, for the host, $\sim 1.5$ mag brighter than for the PSF star fit. $H \sim 16$ corresponds to $\sim 6 \%$ of the 13.05 mag of the QSO nucleus.

Again the QSO residual shows substantial structure as reported in Section 2.2.1. It consists of nested rings of positive and negative flux, typical signs of a close but different width between the PSF we use and the actual one. The bulk of structure is contained in the innermost 0 .'.5 radius and contains $2 / 3$ of the residual flux. The remaining residual of $2 \%$ of the total flux outside this radius is again insignificant, and no main body of the host galaxy co-centered with the quasar is found which satisfies our significance criterion. Going back to the PSF residuals that we quantified earlier on, we detect no co-centered host galaxy at a level above $3 \%$ of the flux of the quasar nucleus, corresponding to an upper limit of $H=16.9$.

However, after removal of the point source, a feature becomes clearer, what we dub the "NE-extension." This faint structure extends from the QSO to the NE, and it can be traced starting at the edge of the strong PSF residuals at 0.'6 $(2.5 \mathrm{kpc}) \mathrm{NE}$ of the nucleus (Figures 3 and 4). Some signs of it are already visible in the optical, when going back to the F606W image (Magain et al. 2005, see also Figure 1), but it is much more pronounced in the new $H$-band data compared to the $V$-band. The NE-extension is possibly part of a tidal arm similar to the arm toward the south of the companion, already described by Canalizo \& Stockton (2001), but our $H$-band image shows it to be clearly disjoint from the companion galaxy. Due to its proximity it is very likely associated with the QSO, even though it is clearly not its main body. It is unlikely that the NE-extension is just a gas cloud with SF induced by the radio jet in the system, since it lies at least $50^{\circ}$ from the jet direction (Feain et al. 2007). It is also unlikely a chance superposition of a gas cloud with emission line gas, as seen by Letawe et al. (2008), since the observed $H$-band does not contain any strong enough line. The NE-extension contains non-negligible flux far above the noise of the background and is unaffected by QSO residuals and independent of the PSF used. We estimate its brightness at $H=18.8$ using an aperture encompassing all visible extension outside the QSO nucleus residual. The same region in the ACS image has $V=21.6$, so $(V-H)=2.8$

In summary, we detect no significant host galaxy that is cocentered with the QSO. We conclude this from the size and shape of the residuals underneath the QSO in comparison to the "PSF star minus foreground star" subtraction residuals we discussed above. The NE-extension, however, that can be seen outside of the residuals of the QSO nucleus, is a real and significant emission structure, and it is very likely associated with the main part of the host galaxy.

\subsubsection{Companion Galaxy}

In the ACS $V$ band the companion galaxy located $1^{\prime \prime} .5$ to the SE appears clumpy, with several bright knots as well as lower surface brightness in the center. Canalizo \& Stockton (2001) even call the companion a "collisional ring galaxy." With the NICMOS $H$ band we get a substantially different picture. The galaxy at $H=15.2$ is still asymmetric, with tidal extensions, but contrary to the visible wavelengths it is smooth and shows a pronounced center: clear signs for substantial dust, not distributed smoothly but unevenly and clumpily, with concentration toward the center that only shows up in the optical (Figure 5). The complexity of the companion is manifested in that there is no good description with neither one or two Sersic components, when the azimuthal shape is restricted to ellipses. The Sersic index of the companion is around $n=2$ for a single Sersic component, and $n<1$ if two Sersic components are used. Taken at face value, both cases point to a more disk- than bulgelike companion, but a substantial fraction in flux is contained in the non-symmetric distorted part of the companion-and this should be the main description of the companion. More complex descriptions were put forward, with either a proposed additional faint AGN hosted by the companion galaxy (Letawe et al. 2009), explosive quasar outflows (Lipari et al. 2009), or quasar-induced SF (Elbaz et al. 2009).

\section{DISCUSSION}

\subsection{Where is the ULIRG?}

There was substantial confusion about the source for the ULIRG-strength IRAS MIR and FIR emission in the literature. From the uncorrected [O II] line flux a star formation rate (SFR) of only $1 M_{\odot} \mathrm{yr}^{-1}$ can be inferred (Kim et al. 2007). Magain et al. (2005) still assign the ULIRG emission to the companion galaxy due to its Balmer decrement which yields non-negligible dust extinction, while Kim et al. (2007) note that the corrected SFR would still be below $10 M_{\odot} \mathrm{yr}^{-1}$. This number is in strong disagreement with a SFR up to $\sim 800 M_{\odot} \mathrm{yr}^{-1}$ inferred from 


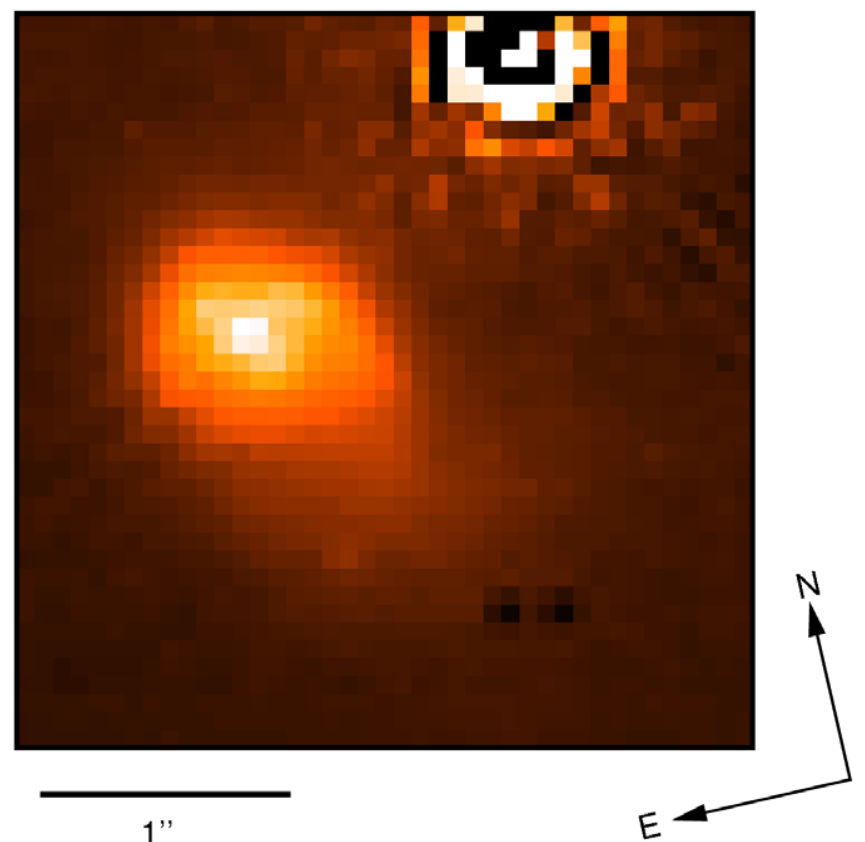

Figure 5. Zoom on the companion galaxy. As a difference to the $I$-band (Figure 1) the galaxy has a pronounced peak of emission and no ring. The light in the optical is obviously attenuated by dust, very strongly in the center where the dust is optically thick, less in the outer regions. Image size is $3^{\prime \prime}$ on the side.

total IR luminosity or $370 M_{\odot} \mathrm{yr}^{-1}$ from $\mathrm{CO}$ (Papadopoulos et al. 2008).

The new NICMOS images show that the stars in the companion galaxy are not distributed in a ring, but the surface brightness increases smoothly towards the center (Figure 5), and that optically thick dust creates the ring-like structure in the optical ACS images (Figure $1(\mathrm{a}+\mathrm{b})$ ). This means that at optical wavelengths only information from the less extincted outer regions of the galaxy as well as the surface of the strongly extincted central regions is seen. UV-based SFRs must therefore dramatically underestimate the true SFRs when corrected with dust extinction estimated from (also optical wavelength) Balmer decrements.

The actual scale of the uncertainty in $A_{V}$, the optical extinction correction, can be estimated by comparing $A_{V}$ estimates from Balmer lines and Paschen/Bracket lines in other ULIRGs. Dannerbauer et al. (2005) studied five ULIRGS for which they estimated $A_{V}$ both from $\mathrm{H}_{\alpha} / \mathrm{H}_{\beta}$ as well as from $\mathrm{Pa} \alpha / \mathrm{Br} \gamma$. NIRderived values for $A_{V}$ were in every case significantly larger, ranging from factors of $\sim 1.16$ to $\sim 10$ (mean 4.0 ) times higher. As this factor does not scale in any way with the optical $A_{V}$ estimate, but only with the NIR estimate, we cannot determine a correction for HE 0450-2958.

When starting out with the redshifted [O II]-line at $\lambda 3727$ and $A_{3727}=A_{V} \times 1.57$ like Kim et al. (2007), and the correction factors from Dannerbauer et al. (2005), a huge range of possible SFRs arises. An average of the two lowest correction values from Dannerbauer et al. (2005) of 1.16 and 1.65 means $A_{V} \sim 2.1$, $A_{3727} \sim 3.3$, or corrected SFRs of $21 M_{\odot} \mathrm{yr}^{-1}$. Using their mean correction factor of $\sim 4$ would lead to $A_{3727} \sim 9.4$ or $>5000 M_{\odot} \mathrm{yr}^{-1}$. So already a number below the mean correction $\left(A_{V} \sim 3\right)$ would make these numbers consistent with FIRemission-based SFR estimates. This directly shows that optical/ UV line-emission-based SFRs as used by Kim et al. (2007) cannot at all be used to constrain the true SFR of ULIRGs and does not provide an argument against strong star formation in the companion.

Papadopoulos et al. (2008) approximated the IRAS IR SED with a two-component blackbody model and found a cool component $T_{\text {dust }}^{\text {cool }}=47 \mathrm{~K}$, dust mass $M_{\text {dust }}^{\text {cool }} \sim 10^{8} M_{\odot}$ and $L_{\mathrm{FIR}} \sim 2.1 \times 10^{12} L_{\odot}$, and a warm component with $T_{\text {dust }}^{\text {warm }}=$ $184 \mathrm{~K}, M_{\text {dust }}^{\text {warm }} \sim 5 \times 10^{4} M_{\odot}$, and $L_{\text {MIR }} \sim 2.6 \times 10^{12} L_{\odot}$. We can now for the first time spatially localize the warm component from the detection of the single $11.3 \mu \mathrm{m}$ point source with VISIR to be coincident with the position of the QSO nucleus. Since the measured flux density is consistent with a warm component having the previously known $12 \mu \mathrm{m}$ IRAS flux density, we conclude that the QSO nucleus itself already is a ULIRG-level emitter, but with a warmer component compared to SF.

For localizing SF in the system, there are two recent new data sets available; radio data from Feain et al. (2007) and the CO maps by Papadopoulos et al. (2008). While the radio maps do not set strong constraints when trying to exploit the radio-FIR relation to assign a location for the FIR emission, the $\mathrm{CO}$ data are more powerful: at least the bulk, possibly all of molecular gas and thus SF activity is located in the companion galaxy.

We can add two further constraints from our NICMOS and VISIR images. Both the MIR SED of the system (Figure 2) as well as an extrapolation from the $H$ band are consistent with an Arp220-like SF, while ruling out milder, M82-like conditions. In the latter case the companion would have to be visible in our observed $11.3 \mu \mathrm{m}$ image, but it is absent (Figure 1). Together with the dense and clumpy dust geometry of the companion when comparing optical and NIR morphology, it becomes clear that the companion is responsible for most, if not all, of the $370 M_{\odot} \mathrm{yr}^{-1} \mathrm{SF}$.

If we follow the 5:1 CO detection significance for the companion given by Papadopoulos et al. (2008), this means that as a minimum $5 / 6=83 \%$ of $\mathrm{CO}$ are located in the companion and thus also $\geqslant 83 \%$ of the SF and FIR emission. This number converts into an integrated IR luminosity of $L_{\mathrm{FIR}} \geqslant 1.75 \times 10^{12} L_{\odot}$, so the companion also qualifies as a ULIRG.

While the presence of very strong SF in the companion is clear now, its trigger is a priori not so clear. The most probable solution is merger-induced SF, so the system would be a classical ULIRG_-just with a non-standard geometry-but there is room for a radio-jet-induced effect as well. One of the lobes of the jets from the QSO is located directly at the companion position. If and how much this contributes to SF in the companion still needs to be quantified.

\subsection{Host Galaxy Detection}

With the companion identified as the main star former, we get limits from the $\mathrm{CO}$ that less than one-sixth of the total cool dust is located within the putative host galaxy. Thus, onesixth of the FIR-inferred SFR by Papadopoulos et al. (2008) of $\mathrm{SFR}=1.76 \times 10^{10}\left(L_{\mathrm{IR}} / L_{\odot}\right) M_{\odot} \mathrm{yr}^{-1}$ correspond to an upper limit of $62 M_{\odot} \mathrm{yr}^{-1}$. This leaves room for a non-negligible amount of SF in the host galaxy, but is also an upper limit. ${ }^{7}$

\footnotetext{
Note that for the galaxy-scale SFRs around QSO nuclei the dust can be heated by a mix of stellar emission as well as energy from the AGNs. In this sense, the $47 \mathrm{~K}$ found for the cool dust component of HE 0450-2958 agrees well with the mean SF-heated dust around higher- $z$ QSOs (also $47 \mathrm{~K}$, Beelen et al. 2006), and can be composed of intrinsically cooler dust (20-30 K) plus AGN heating. This temperature could thus be a hint that indeed a part of this cool dust component is located in the QSO host galaxy and not in the companion.
} 
If we assume the host galaxy to have a mix of old and young stellar population as we find for other QSO host galaxies at these redshifts (Jahnke et al. 2004a; Letawe et al. 2007), we can convert this to an expected $H$-band flux. If the host galaxy had the same population mix as Canalizo \& Stockton (2001) modeled for the companion galaxy ${ }^{8}-95.5 \%$ of a $10 \mathrm{Gyr}$ old population with 5 Gyr e-folding SFR timescale plus $4.5 \%$ of a $128 \mathrm{Myr}$ young population - this SFR upper limit would translate into an expected NIR magnitude of 1.75 mag fainter than the companion or $H \geqslant 16.95$. The combined color and $K$-correction term is $V-H_{\mathrm{z}=0.285}=1.66$, and changes by only about $\pm 0.3 \mathrm{mag}$ for a pure old $(10 \mathrm{Gyr})$ or young $(100 \mathrm{Myr})$ population. So they are rather insensitive to the exact choice of stellar population. However, this limit will get brighter if the host galaxy contained less dust-by about 0.3 mag per magnitude decrease in $A_{V}$.

With that in mind, this limit is not more stringent than the limit from NICMOS itself: no significant main host-galaxy body is found after PSF removal (Section 3.2.1) and so an upper limit from the NIR decomposition of $H=16.9$ applies for a host galaxy co-centered with the quasar nucleus. We, therefore, conclude that the current upper limit from NICMOS lies at around $H \sim 16.9$. This is consistent with the CO/FIR limits.

How do these numbers relate to the current upper limit for a co-centered host galaxy from the optical HST data? We convert our $H$-band limit to absolute $V$-band magnitudes with again the assumption of the host galaxy having the same stellar population mix as the companion. In the conversion to $M_{V}$ we assume two different values for dust extinction, (1) $A_{V}=0$, motivated by the nearly dust-free line of sight to the QSO nucleus, and (2) a moderate $A_{V}=1$ (corresponding to $A_{H(\mathrm{z}=0.285)} \sim 0.29$ ). This yields host-galaxy upper limits of $M_{V}>-21.25$ and $>-22.55$, for the cases (1) and (2), respectively. If we convert the Magain et al. (2005) upper limits to our $h=0.7$ cosmology and assume the same stellar population and dust properties, we receive $M_{V}>-20.6$ and $>-21.6$, respectively. We note here that this corresponds to a detection limit of only $1.5 \%$ of the total quasar flux in the optical. This factor of 2 is owed to the better determined PSF in the ACS images. This allows Magain et al. to set somewhat stricter upper limits for a nucleus co-centered host-galaxy component, particularly if a low dust extinction is present.

Concerning lower limits to the host galaxy, the NE-extension (Figure 4) is a structure of real emission that can be traced toward the QSO from $\sim 1^{\prime \prime} .5$ to a radius of 0 .' 6 , where the region of substantial PSF residuals begins. We cannot say for sure whether it continues further inward from this position. Signs of this structure are visible in the ACS $V$-band (see Figure 1, left column) but it is not clear whether the more compact region only $\sim 0^{\prime} .2 \mathrm{NE}$ of the nucleus in ACS image is real or an artifact of the deconvolution process. We measured the $(V-H)$ color to be 2.8 outside this region, which is consistent with a stellar population of intermediate age. In the dust-free case, this color corresponds to a $\sim 2.1$ Gyr old single stellar population (Bruzual $\&$ Charlot 2003, solar metallicity), for $A_{V}=1.0$ to an age of 800 Myr. This is consistent with stellar material from a host galaxy, e.g., tidally ejected disk stars.

We conclude that with its spatial detachment from the companion galaxy this NE-extension is likely a part of the host galaxy, possibly as a tidal extension, but its vicinity to the QSO

\footnotetext{
8 Canalizo \& Stockton (2001) used optical spectra only. With the optically thick dust now detected we have to restrict their diagnosis to mainly the outer parts and surface of the companion. The population mix there might be identical to the core of the companion, but it does not necessarily have to.
}

makes other interpretations less likely. With this interpretation, we receive an $H \leqslant 18.8$ lower limit for the host, corresponding to $M_{V}<-20.4\left(A_{V}=0\right)$ or $<-20.7\left(A_{V}=1\right)$. If we include this off-center emission to the upper limit of a cocentered host galaxy, we obtain a total host-galaxy upper limit of $M_{V}>-21.2$ and -22.0 . We thus bracket the host-galaxy luminosity in the $V$ band by 0.8 and $1.3 \mathrm{mag}$ or factors of $\sim 2$ and $\sim 3.5$, respectively.

Formally, the $\mathrm{CO}$ detection significance and NICMOS give the same limit on a SFR of up to $\sim 60 M_{\odot} \mathrm{yr}^{-1}$. If we take into account the stricter ACS $V$-band limits of $M_{V}>-20.6$ and $>-21.6$, depending on dust cases (1) and (2), these are fainter by 1.3 and 0.6 mag than the $\mathrm{CO}$-predicted magnitudes. Inversely, these reduce the upper limits on SF to 18 and $35 M_{\odot}$ $\mathrm{yr}^{-1}$, respectively. Beyond $A_{V}=2 \mathrm{mag}$ the CO and NICMOS limits again become the most stringent. This means that we cannot rule out dust obscuration in the host galaxy. At the same time the dust-free line of sight to the quasar nucleus is a strong argument against large amounts of dust, unless a very special geometrical configuration is invoked, while the warm ULIRG emission from the QSO points to dust in the very central few $100 \mathrm{pc}$. Only better CO limits or a detection of the host galaxy in the NIR will be able to finally resolve this matter.

\subsection{Black Hole Mass, Galaxy Luminosity, and the NLSyl Angle}

BH mass estimates for HE 0450-2958 vary significantly through the literature. The original $8 \times 10^{8} M_{\odot}$ (Magain et al. 2005) were revised later to a substantially lower value of $4 \times 10^{7}$ (Letawe et al. 2007). Both values are virial estimates based on $\mathrm{H} \beta$ width, but while narrow and broad components were separately measured in the former study, the FWHM of the whole line was used in the latter. This revised value is consistent with the independent virial estimate of $6-9 \times 10^{7}$ by Merritt et al. (2006), and even with an estimate from X-ray variability, $2_{-1.3}^{+7} \times 10^{7}$ (Zhou et al. 2007). Since the virial estimates agree now, we will adopt the range $4-9 \times 10^{7} M_{\odot}$ for the BH mass.

Merritt et al. (2006) noted the rather narrow broad emission lines of HE 0450-2958 and suggested that it should actually be viewed not as a standard QSO but as a higher- $L$ analog of local NLSy1s. If we compare HE 0450-2958 with estimates from the literature (Grupe \& Mathur 2004; Ohta et al. 2007), we find that HE 0450-2958 is consistent with the high BH mass end of the known NLSy1 distribution and does not need to constitute a new "higher- L NLSy1 analog" class of its own. But is it consistent regarding other properties as well?

Morphologically, NLSy1 are mostly spirals, often barred, mostly not strongly disturbed (Ohta et al. 2007). Since galaxies have increasing bulge mass with increasing $\mathrm{BH}$ mass it is not clear which structural properties to expect and if a merging system like this is consistent with the properties of the local, lower-mass NLSy1 population.

There is even a debate on how different NLSy1 actually are from normal Seyferts. Recent studies show smaller $\mathrm{BH}$ mass differences between normal broad-line Sy1 and NLSy1 when using line dispersions instead of FWHM (Watson et al. 2007), although a difference might remain. If galaxies with potentially core outflow-affected lines are considered separately, NLSy1 share the same $M_{\mathrm{BH}}-\sigma_{\text {bulge }}$ relation with BLSy1, but their accretion rates are confirmed as lying often close to the Eddington limit (Komossa \& $\mathrm{Xu}$ 2007). If we compute the HE 0450-2958 accretion rate-as derived from the $V$-band absolute magnitude of the quasar nucleus $\left(M_{V}=-25.75\right.$, 


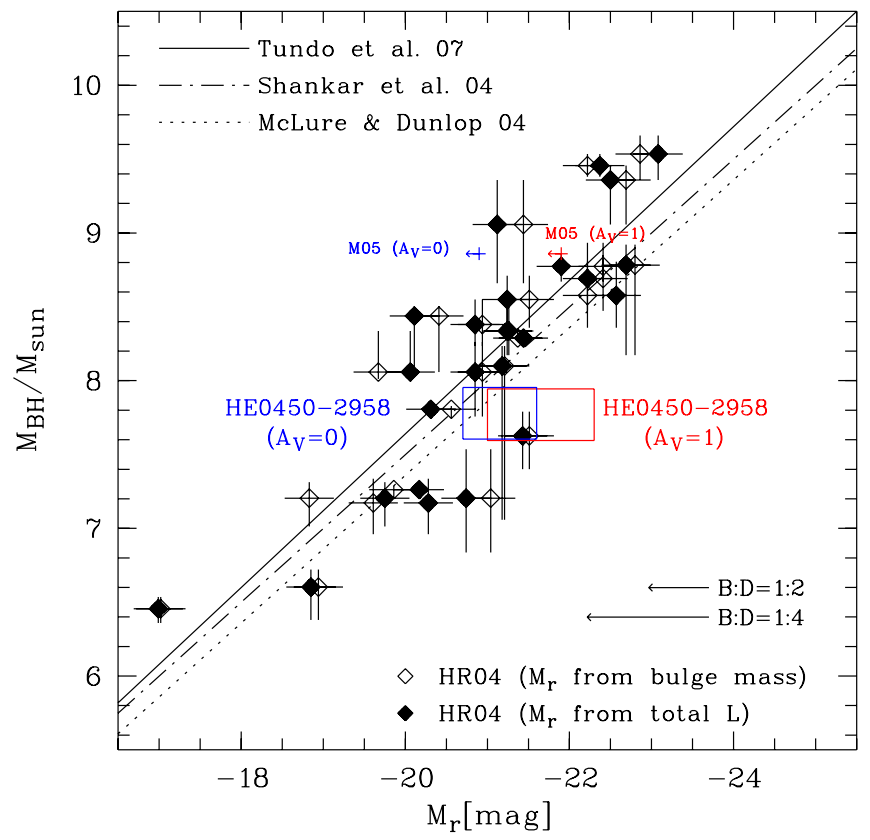

Figure 6. $M_{\mathrm{BH}}-L_{\text {bulge }}$ relation for inactive galaxies in the local universe as presented by Tundo et al. (2007), with data from Häring \& Rix (2004), Shankar et al. (2004) and McLure \& Dunlop (2004) (black lozenges and lines). Overplotted are the upper limits for the host galaxy of HE 0450-2958 for the dust-free case by Magain et al. (2005) from $V$-band imaging (small blue arrow) and with an $A_{V}=1$ added (small red arrow), with their original $\mathrm{BH}$ estimate, converted to our cosmology. The blue and red rectangles show the range for $\mathrm{BH}$ mass estimates and our new lower limits for the (total) galaxy luminosity from NICMOS and new upper limits based on the (still better constrained) optical $H S T$ data. Note: here we combined the off-center flux lower limit (NICMOS $H$-band) with the upper limit for a co-centered host galaxy (ACS $V$-band) for a total upper limit. The arrows to the bottom right show the conversion of our $L_{\text {galaxy }}$ limits to $L_{\text {bulge }}$ limits for bulge-to-disk ratios of 1:2 and 1:4. Both the dust-free as well as the $A_{V}=1$ dust case show a galaxy that is absolutely consistent with the $\mathrm{BH}$ mass, even if the bulge-to-disk ratio is accounted for.

recomputed from the $H S T /$ ACS data with updated AGN color and $K$-correction) and a bolometric correction of $B C_{V} \sim 8$ (Marconi et al. 2004; Elvis et al. 1994)—in relation to its Eddington accretion rate, we obtain from $M_{\mathrm{BH}}=6.5 \pm 2.5 \times$ $10^{7} M_{\odot}$ a super-Eddington accretion rate of $L / L_{\text {Edd }}=6.2_{-1.8}^{+3.8}$. This is consistent with high Eddington ratios observed for NLSy1 (Warner et al. 2004; Mathur \& Grupe 2005).

With the new data and an explicit assumption/interpretation that the NE-extension is indeed associated with the host galaxy, we can for the first time present a BH mass for HE 0450-2958 and bracketing limits for its host-galaxy luminosity. We can thus place HE 0450-2958 on the $M_{\mathrm{BH}}-L_{\text {bulge }}$ relation of active and inactive galaxies, with more than just an upper limit for galaxy luminosity. In Figure 6, we show data from Häring \& Rix (2004) and others, as collected by Tundo et al. (2007). We overplotted the limits on HE 0450-2958 for the two assumptions of dust attenuation strength (Section 4.2). This shows that even when applying a sensible conversion factor of 1 to $1 / 4$ (up to $1.5 \mathrm{mag}$ ) to convert from total to bulge luminosity, the host of HE 04502958 will be a perfectly normal galaxy in this parameter space, with a luminosity around the knee of the galaxy luminosity function, $L \sim L^{*}$.

Contrary to the claim by Magain et al. (2005) it does not deviate substantially from the local $M_{\mathrm{BH}}-L_{\text {bulge }}$ relation for normal inactive local massive galaxies, mainly due to the revised mass estimate for the $\mathrm{BH}$. However, this also means that HE 0450-2958 does not show a $M_{\mathrm{BH}} / L_{\text {bulge }}$ different from local broad-line AGNs, consistent with being a NLSy1-analog if the Komossa \& $\mathrm{Xu}$ (2007) result is taken as a base.

With the normal $M_{\mathrm{BH}} / L_{\text {bulge }}$ ratio and the fact that we can now rule out huge amounts of obscuring dust around the QSO nucleus, the most likely explanation for the evasive host galaxy is indeed a high $L / L_{\text {Edd }}$ accretion rate system-a NLSy1 at the high-mass end of the normal NLSy1 population. With the current evidence Occam's Razor favors this explanation over more exotic scenarios as the ejection of the QSO's BH in a three-body interaction or a gravitational recoil event involving the companion galaxy (e.g., Hoffman \& Loeb 2006; Haehnelt et al. 2006; Merritt et al. 2006; Bonning et al. 2007). However, these scenarios are formally not ruled out even if the upper limit can be pushed down by another $\sim 5$ magnitudes. All evidence combined is consistent with a system of a QSO with ULIRGsize IR emission, residing in an $L^{*}$ host galaxy that is in the process of colliding with a substantially more luminous and possibly more massive companion ULIR-galaxy. ${ }^{9}$ Much deeper high-resolution NIR imaging with a well controlled PSF is the best way to finally find and trace the host galaxy (bulge) component of HE 0450-2958 that is predicted here to be cocentered with the QSO nucleus and to estimate its luminosity and mass directly.

\subsection{Black Hole-Galaxy Coevolution}

Given the $\mathrm{BH}$ mass and Eddington ratio the accretion rate of the $\mathrm{BH}$ is $1.4 M_{\odot} \mathrm{yr}^{-1}$. At the same time Papadopoulos et al. (2008) derived a SFR from CO of $370 M_{\odot} \mathrm{yr}^{-1}$, predominantly in the companion galaxy. Applying a correction factor of 0.5 for mass returned to the interstellar matter by stellar winds, the stellar mass growth of the whole HE 0450-2958 system from $\mathrm{SF}$ is $185 M_{\odot} \mathrm{yr}^{-1}$. The ratio of $\mathrm{BH}$ accretion and stellar mass growth is then $12 / 185=6.5 \%$, which is substantially higher than the $M_{\mathrm{BH}} / M_{\text {bulge }}$ relation for local galaxies of $0.14 \%$ (Häring \& Rix 2004).

We can conclude the following: if activity timescales are identical for $\mathrm{SF}$ and $\mathrm{BH}$ accretion, this system grows in $\mathrm{BH}$ mass much more rapidly than the bulge is required to grow to keep the system on the $M_{\mathrm{BH}} / M_{\text {bulge }}$ relation. This is not possible, since the SF is taking place in the companion and not the host galaxy. So in any case a potential maintenance of the relation for this system, if actually true, needs to be seen as an integral over more than several $10^{8} \mathrm{yr}$.

On the other hand, a gas consumption timescale of $9.5 \times$ $10^{7} \mathrm{yr}$ - if we divide the $\mathrm{H}_{2}$ masses and $\mathrm{SF}$ rates derived by $\mathrm{Pa}-$ padopoulos et al. (2008) and account for 50\% mass recyclingis possibly longer than the luminous quasar accretion phase. This would add to the requirement, that processes like the tidal forces of the galaxy interaction redistribute mass, adding stars to the bulge of the host galaxy. These were to the larger extent already preexisting in the host galaxies disk or the companion before the interaction and not created only now. The "coevolution" of the host galaxy and its BH in HE 0450-2958 is clearly a two-part process: the build-up of stellar mass and the build-up of $\mathrm{BH}$ and bulge mass. The former will take place on timescales of $>1$ Gyr through SF, the latter two can "coevolve" if seen

\footnotetext{
9 It is interesting to note that the "companion" is close to a factor of 10 more luminous than the host galaxy. With all uncertainties included it would still appear as if the typical mass ratio upper limit of 1:3 for the merging galaxies in a ULIRG system (Dasyra et al. 2006) were exceeded here. However, when using the dynamical masses from Papadopoulos et al. (2008) to predict a BH mass in the host galaxy consistent with the Häring \& Rix (2004) relation, we get a merger mass ratio of $1: 1$ or $1: 2$.
} 
as an average over timescales of longer than the $\mathrm{BH}$ accretion lifetime, and a few dynamical timescales for redistribution of stellar orbits of, say, $<500 \mathrm{Myr}$.

\subsection{How Many HE 0450-2958s are There?}

HE 0450-2958 is an unusual object. AGNs in ULIRGs are common, but AGNs right next to ULIRGs are not, particularly not luminous QSOs with inconspicuous host galaxies next to extreme star formers. So, is HE 0450-2958 one of a kind, or was it just the scarceness of IR imaging with $1^{\prime \prime}$ resolution and high-resolution $\mathrm{CO}$ maps that prevents us from finding similar objects en masse?

In the higher redshift universe there was a recent report of a very similar system (Younger et al. 2008). LH850.02 at $z=3.3$ is the brightest submillimeter galaxy in the Lockman hole. Using the Submillimeter Array (SMA), the authors find two components of which one is a ULIRG with intense SF, while the other component likely harbors an AGN. At $z>2$ however, objects like this might be quite common, since merging rates and gas reservoirs were much larger than today. If there existed a substantial number of similar systems at low redshifts, this would allow to study mechanisms of the high-redshift universe at much lower distances.

We try to estimate the frequency of such systems in the local universe using the three morphologically best studied samples of quasars at $0.05 \lesssim z<0.43$. We deliberately use optically selected quasars only, as they have no bias with respect to frequency of merger signatures or extreme SFRs as IRselected samples have by construction. In this way, statements about the general population are possible. Jahnke et al. (2004a) investigated a volume-limited and complete sample of 19 luminous QSOs out to $z=0.2$. While at least five of these QSOs are seen in intermediate and late stages of major mergers, only one, HE $1254-0934$, is a likely ULIRG,${ }^{10}$ as determined from its IRAS fluxes. It is also among the most distorted systems, with a companion at $\sim 1^{\prime \prime}$ distance from the QSO nucleus. The companion is more luminous than the host galaxy, and shows a substantial tidal tail. It looks remarkably similar to HE 04502958.

The two other samples are not volume-limited samples, so the selection function is unclear-except that these quasars stem from either optical or radio surveys, but not the IR. Floyd et al. (2004) studied the morphologies of two intermediate- and high-luminosity samples of ten radio-quiet and seven radioloud quasars at $0.29<z<0.43$, using HST-imaging data. Only one of their 17 quasars shows a distorted geometry similar to HE 0450-2958 (1237-040 at $z=0.371)$ but there exists no information about the total IR emission or SFRs. The IRAS flux limits of $200 \mathrm{mJy}$ are equivalent to upper limits of $L_{\mathrm{ir}} \sim 6 \times 10^{12} L_{\odot}$ at $z=0.37$. ULIRG-strength emission for $1237-040$ could have gone unnoticed by IRAS.

A recent study by Kim et al. (2008) determined the morphologies of $45 H S T$-archived quasars at $z<0.35$. It has one object in common with Floyd et al. (2004) and three objects with Jahnke et al. (2004a). Of their sample, three other objects (HE 0354-5500, PG 1613+658, and PKS 2349-01) are clearly merging with a nearby companion, and are likely ULIRGs as judged from their IRAS fluxes. However, only in the case of HE 0354-5500 the quasar and companion are still well separated and their envelopes have not yet merged into a common

\footnotetext{
10 This is a borderline case because it will fall slightly below or above the ULIRG definition limit depending on if we include upper limits in 12 and $25 \mu \mathrm{m}$ or assume the flux to be zero.
}

halo. The two other cases are in a very late merger state and SF will likely occur all over the system.

This adds up to only $\leqslant 3 / 77$ QSOs to possibly be HE 0450 2958 -like in the three samples combined. At $\leqslant 4 \%$, such systems are indeed rare in the local universe. These three quasars, however, should be investigated in more detail. It needs to be tested how strong their SF actually is, where in the system it is localized, and if the separated companion is in any way connected to the AGN-fueling. If a similar situation as for HE 0450-2958 is found, the result can set strong constraints on the ULIRG-AGN evolutionary scenario (Sanders \& Mirabel 1996) and the creation mechanisms of AGNs at high redshifts. It can contribute to answering the question whether SF-ULIRG activity in AGN systems is an indicator of a specific mechanism of AGN fueling. Or, if these are just the most gas-rich mergertriggered AGN systems at the top end of SFRs, with a continuous sequence toward less gas-rich merger-triggered AGN systems. The merging-AGN fueling mechanism could be identical from ULIRGs down to the Seyfert regime, where at some point secular mechanisms become more dominant. Lower SFR systems could just be the consequence of lower gas mass, but this might only mildly impact the-much smaller-AGN fuelling rate.

\section{CONCLUSIONS}

With new NIR and MIR images to spatially resolve the HE 0450-2958 system, and in the light of previously existing data, we find:

1. The companion galaxy is covered in optically thick and unevenly distributed dust. This makes it appear as a collisional ring galaxy in the optical, but intrinsically it is smooth and has smooth NIR emission increasing toward a pronounced center. The SF in the companion is similar to the strong starburst Arp220, while softer M82-like SF is ruled out. This can reconcile the SFR estimates from the optical and FIR. The companion is a SF powered ULIRG.

2. Our MIR image confirms a single warm dust point source at the location of the QSO nucleus. This supports a two component dust SED with the warm component fully associated with the QSO nucleus, which is an AGNpowered ULIRG.

3. A dust-free line of sight to the quasar nucleus is evidence that the host galaxy is not obscured by large amounts of dust. However, the ULIRG-strength warm IR emission by the nucleus and the upper limit on SF in the host galaxy of substantial $60 M_{\odot} \mathrm{yr}^{-1}$ leave room for dust.

4. With $H \geqslant 16.9$, the current NICMOS images do not set stronger upper limits on the host galaxy of HE 0450 2958. The $V$-band, $H$-band, and CO-constraints give $M_{V} \geqslant$ -21.2 to $M_{V} \geqslant-22.0$ depending on the assumed dust masses.

5. Flux in the NE-extension of $H=18.8$ is likely associated with the QSO's host galaxy. It corresponds to a first lower limit of $M_{V}<-20.4$ for the host galaxy. With a BH of $\sim 6.5 \pm 2.5 \times 10^{7} M_{\odot}$, an accreting rate of $12 M_{\odot} \mathrm{yr}^{-1}$ equal to super-Eddington accretion, $L / L_{\mathrm{Edd}}=6.2_{-1.8}^{+3.8}$, the host galaxy is consistent with the $M_{\mathrm{BH}}-M_{\text {bulge }}$ relation for normal galaxies. It is also consistent with HE 0450-2958 being a NLSy1 at the high end of the known BH mass distribution. The reason for the high accretion rate is unclear but could be connected to HE 0450-2958 being in an early stage of merging with its gas-rich companion. A more exotic explanation for the system is currently not required by any 
data, but can in the end only be ruled out with much deeper, high-resolution NIR images to find the main body and bulge of the host galaxy.

6. If host galaxy and BH in HE 0450-2958 are co-evolving according to the local $M_{\mathrm{BH}}-M_{\text {bulge }}$ relation, it has to occur over longer timescales ( $\leqslant 500 \mathrm{Myr}$ ) and/or the mass growth for the bulge is predominantly not caused by the current SF in the system, but by redistribution of preexisting stars.

7. A constellation as in the HE 0450-2958 system with separate locations of QSO nucleus and strongly starforming ULIRG companion might be common at $z>2$ where gas masses and merger rates were higher, but at a fraction of $\leqslant 4 \%$ it is extremely rare in the local universe.

The authors thank E. F. Bell, A. Martínez Sansigre, H. Dannerbauer, E. Schinnerer, K. Meisenheimer, F. Courbin, P. Magain, and H.-R. Klöckner for very fruitful discussions and helpful pointers.

Based on observations made with ESO Telescopes at the Paranal Observatory under programme ID 276.B-5011. Also based on observations made with the NASA/ESA Hubble Space Telescope, obtained at the Space Telescope Science Institute, which is operated by the Association of Universities for Research in Astronomy, Inc., under NASA contract NAS 526555 . These observations are associated with program \#10797. This research has made use of the NASA/IPAC Extragalactic Database (NED).

K.J. acknowledges support through the Emmy Noether Programme of the German Science Foundation (DFG) with grant number JA 1114/3-1. A.B. is funded by the Deutsches Zentrum für Luft- und Raumfahrt (DLR) under grant 50 OR 0404. VC, Research Fellow, thanks Belgian Funds for Scientific Research. This work was also supported by PRODEX experiment arrangement 90312 (ESA and PPS Science Policy, Belgium).

Facilities: ESO VLT (VISIR), HST (NICMOS).

\section{REFERENCES}

Bahcall, J. N., Kirhakos, S., \& Schneider, D. P. 1994, ApJ, 435, L11 Bahcall, J. N., Kirhakos, S., \& Schneider, D. P. 1995, ApJ, 450, 486 Beelen, A., Cox, P., Benford, D. J., Dowell, C. D., Kovács, A., Bertoldi, F., Omont, A., \& Carilli, C. L. 2006, ApJ, 642, 694

Bonning, E. W., Shields, G. A., \& Salviander, S. 2007, ApJ, 666, L13

Boyce, P. J., et al. 1996, ApJ, 473, 760

Bruzual, G., \& Charlot, S. 2003, MNRAS, 344, 1000

Canalizo, G., \& Stockton, A. 2001, ApJ, 555, 719

Chantry, V., \& Magain, P. 2007, A\&A, 470, 467

Dannerbauer, H., Rigopoulou, D., Lutz, D., Genzel, R., Sturm, E., \& Moorwood, A. F. M. 2005, A\&A, 441, 999

Dasyra, K. M., et al. 2006, ApJ, 638, 745 de Grijp, M. H. K., Lub, J., \& Miley, G. K. 1987, AApSS, 70, 95

Elbaz, D., Cesarsky, C. J., Chanial, P., Aussel, H., Franceschini, A., Fadda, D., \& Chary, R. R. 2002, A\&A, 384, 848

Elbaz, D., et al. 2009, A\&A, submitted

Elvis, M., et al. 1994, ApJS, 95, 1

Feain, I. J., Papadopoulos, P. P., Ekers, R. D., \& Middelberg, E. 2007, ApJ, 662 , 872

Floyd, D. J. E., Kukula, M. J., Dunlop, J. S., McLure, R. J., Miller, L., Percival, W. J., Baum, S. A., \& O'Dea, C. P. 2004, MNRAS, 355, 196

Groenewegen, M. A. T., et al. 2002, A\&A, 392, 741

Grupe, D., \& Mathur, S. 2004, ApJ, 606, L41

Haehnelt, M. G., Davies, M. B., \& Rees, M. J. 2006, MNRAS, 366, L22

Häring, N., \& Rix, H.-W. 2004, ApJ, 604, L89

Hoffman, L., \& Loeb, A. 2006, ApJ, 638, L75

Hopkins, P. F., Hernquist, L., Cox, T. J., Di Matteo, T., Robertson, B., \& Springel, V. 2006, ApJS, 163, 1

Jahnke, K., Kuhlbrodt, B. \& Wisotzki, L. 2004a, MNRAS, 352, 399

Jahnke, K., et al. 2004b, ApJ, 614, 568

Kim, M., Ho, L. C., Peng, C. Y., Barth, A. J., Im, M., Martini, P., \& Nelson, C. H. 2008, ApJ, 687, 767

Kim, M., Ho, L. C., Peng, C. Y., \& Im, M. 2007, ApJ, 658, 107

Komossa, S., \& Xu, D. 2007, ApJ, 667, L33

Letawe, G., Magain, P., Chantry, V., \& Letawe, Y. 2009, MNRAS, 396, 78

Letawe, G., Magain, P., \& Courbin, F. 2008, A\&A, 480, 69

Letawe, G., Magain, P., Courbin, F., Jablonka, P., Jahnke, K., Meylan, G., \& Wisotzki, L. 2007, MNRAS, 378, 83

Lipari, S., et al. 2009, MNRAS, in press (arXiv:0901.3292)

Low, F. J., Cutri, R. M., Huchra, J. P., \& Kleinmann, S. G. 1988, ApJ, 327, L41

Low, F. J., Cutri, R. M., Kleinmann, S. G., \& Huchra, J. P. 1989, ApJ, 340, L1

Magain, P., Courbin, F., \& Sohy, S. 1998, ApJ, 494, 472

Magain, P., Letawe, G., Courbin, F., Jablonka, P., Jahnke, K., Meylan, G., \& Wisotzki, L. 2005, Nature, 437, 381

Marconi, A., Risaliti, G., Gilli, R., Hunt, L. K., Maiolino, R., \& Salvati, M. 2004, MNRAS, 351, 169

Mathur, S., \& Grupe, D. 2005, ApJ, 633, 688

McLeod, K. K., \& Rieke, G. H. 1995, ApJ, 454, L77

McLure, R. J., \& Dunlop, J. S. 2004, MNRAS, 352, 1390

Merritt, D., Storchi-Bergmann, T., Robinson, A., Batcheldor, D., Axon, D., \& Cid Fernandes, R. 2006, MNRAS, 367, 1746

Ohta, K., Aoki, K., Kawaguchi, T., \& Kiuchi, G. 2007, ApJS, 169, 1

Pantin, E., Vanzi, L., \& Weilenman, U. 2008, in the ESO Astrophysics Symp., The 2007 ESO Instrument Calibration Workshop (Berlin: Springer)

Papadopoulos, P. P., Feain, I. J., Wagg, J., \& Wilner, D. J. 2008, ApJ, 684, 845

Peng, C. Y., Ho, L. C., Impey, C. D., \& Rix, H.-W. 2002, AJ, 124, 266

Sanders, D. B., \& Mirabel, I. F. 1996, ARA\&A, 34, 749

Sérsic, J. 1968, Atlas de Galaxias Australes, Observatorio Astronomico de Cordoba

Shankar, F., Salucci, P., Granato, G. L., De Zotti, G., \& Danese, L. 2004, MNRAS, 354, 1020

Somerville, R. S., Hopkins, P. F., Cox, T. J., Robertson, B. E., \& Hernquist, L. 2008, MNRAS, 391, 481

Tundo, E., Bernardi, M., Hyde, J. B., Sheth, R. K., \& Pizzella, A. 2007, ApJ, 663,53

Warner, C., Hamann, F., \& Dietrich, M. 2004, ApJ, 608, 136

Watson, L. C., Mathur, S., \& Grupe, D. 2007, AJ, 133, 2435

Younger, J. D., et al. 2008, MNRAS, 387, 707

Zhou, X.-L., Yang, F., Lü, X.-R., \& Wang, J.-M. 2007, AJ, 133, 432 